\title{
Ramification des racines nodales primaires du maïs (Zea mays L). Données de structure et de cinétique
}

\author{
MO Jordan 1, D Picard 2, R Trendel 3 \\ 1 INRA, station d'agronomie, BP 91, 84143 Montfavet Cedex; \\ 2 INRA, département d'agronomie, 78850 Thiverval-Grignon; \\ 3 INRA, station d'agronomie, BP 507, 68021 Colmar Cedex, France
}

(Reçu le 11 avril 1991; accepté le 25 novembre 1991)

\begin{abstract}
Résumé - Ce travail a pour but de caractériser les paramètres descriptifs de la structure et de la cinétique de ramification des racines nodales primaires du maïs. Chaque fois que cela a été possible, les déterminations ont eu lieu au champ, sinon en rhizotrons.

Nous avons montré que chaque racine primaire est formée d'une partie ramifiée qui se prolonge en deçà de l'apex, par une «zone nue» a dont la longueur varie de 10 à $19 \mathrm{~cm}$. A la base de la racine, à proximité du point de raccordement à la tige, existe une seconde partie non ramifiée $b$. La longueur de $b$, comprise entre 0,4 et $5,3 \mathrm{~cm}$, se déduit du numéro de l'entre-nœud d'insertion de la primaire (noté $\mathrm{E}$ ), à l'aide d'une régression sur les variables transformées en données logarithmiques.

Les nombres de secondaires (appelées également racines d'ordre 2) par unité de longueur augmentent avec le rang $E$ de la racine primaire porteuse. Ils passent de 6,5 ramifications/cm pour $E_{2}$ à 16,0 pour $E_{7}$. La variabilité sur les mesures, pour un nombre de répétitions important (compris entre 74 et 177) est d'environ $30 \%$. Les taux de ramifications ont tendance à baisser légèrement avec la profondeur et sont significativement différents les 2 années d'étude. Par ailleurs, la proportion de racines d'ordre 2 qui s'allongent au-delà de $3 \mathrm{~cm}$ est relativement faible et significativement plus élevée en 1984 qu'en 1985. Pour ces 2 années, les pourcentages moyens de secondaires longues observés sur les différents entre-nœuds sont respectivement compris entre 20 et $31 \%$ et entre 10 et $25 \%$.

Une primaire se ramifie dès que sa longueur est supérieure à "a $a b »$. L'émission racinaire est localisée dans la zone de rhizogenèse située au-dessous de l'apex et de la zone pillifère. Elle est soumise à un double déterminisme : c'est le nombre de feuilles visibles qui détermine le début de l'émergence secondaire sur les primaires d'un entre-nœud donné. On observe un décalage de 2,2 phyllochrones entre l'émission foliaire et l'apparition des premières secondaires à un entre-nœud donné. Le front d'émission progresse ensuite le long de la primaire en fonction de la vitesse d'élongation de cette dernière.
\end{abstract}

Zea mays L = maïs / structure racinaire / racine secondaire / développement racinaire / nombre de racines

Summary - Secondary ramification of corn (Zea mays L) nodal roots. Kinetics and structural data. The aim of this work is to give an evaluation, during the whole cultural cycle, of the parameters describing corn root secondary ramification. If possible, experimentation has been done in the field, otherwise in root-boxes. Each primary root is formed (as described in figure 1) on a branching zone surrounded by 2 bare parts: "a" located just above the apex and " $\mathrm{b}$ ", at the top of the root. The length of "a", given in figure 2 according to the method used, fluctuates between 10 and $19 \mathrm{~cm}$ and seems to be independent of the insertion number of the primary root (called $\mathrm{E}_{\mathrm{i}}$ ). The length of "b" increases with $\mathrm{E}_{\mathrm{i}}$ and varies from 0.4 to $5.3 \mathrm{~cm}$ as shown by the relation:

$$
\operatorname{Ln} b=1.64 \operatorname{Ln} E_{i}-2.03 \quad r=0.97^{\star * *}
$$

Numbers of secondary roots are given per unit length, ie $\mathrm{cm}$ of primary mother root, in figure 3 for the needle boards and in 4 for the rhizotrons. They increase with $E_{i}$, vary from 6.5 ramification $/ \mathrm{cm}$ for $E_{2}$ to 16.0 Rll/cm for $E_{7}$ and have a tendency to decrease with depth. They also vary significantly between the 2 years (see variance analysis in table II). The variation coefficient between the countings, for an important number of replications which are given in table I varies from 74 to 177 , with an average of $30 \%$. The proportion of secondary roots with an elongation of more than $3 \mathrm{~cm}$ (given in figure 4, according to the depth) is relatively low and significantly higher in 1984 than in 1985. For these 2 years it respectively varies between 20 and $31 \%$ in 1984 and 10 and $25 \%$ in 1985 . For the numbers of elongated roots, the years also have an influence on the standard deviations (or $S D$ ), as is shown in figure 5 which gives the $S D$ versus means for both years. 
Ramifications appear on the primary root as soon as its length is greater than $(\mathrm{a}+\mathrm{b})$. Their emission occurs above the apex and follows a double determinism. Emission beginning at a given internode is a function of the visible leaves (called NF), as follows:

$$
N F=2.20 E_{i}+0.91 ; r=0.99^{* * *} ; i<7
$$

Then the ramification front progresses along the primary root with root elongation. The distance from the primary root basis where secondary ramification occurs is plotted for each internode in figure $6 a$ and $6 b$ versus number of days after sowing and number of visible leaves. However, the emission duration of the first $90 \mathrm{~cm}$ of a primary root (length which can be followed through the rhizotron glass wall) varies widely (table III) with its internode rank.

Zea mays $L$ = maize / root structure / secondary root / root development / root number

\section{INTRODUCTION}

À l'échelle de la parcelle cultivée, la compréhension du fonctionnement du système racinaire nécessite une bonne connaissance de sa structure (Habib et al, 1991) définie par le nombre d'organes, leurs relations dans le temps et dans l'espace (Franquin, 1974). Cependant, nos lacunes en ce domaine sont importantes.

En effet, les travaux entrepris au champ, échelle de référence pour l'agronome, ont souvent été limités à l'étude de l'influence d'une condition de milieu sur un paramètre descriptif de l'enracinement : c'est ainsi que, par exemple, les vitesses d'élongation ont été exprimées en fonction, soit de la température (Blacklow, 1972) soit du potentiel matriciel (Mirreh et Ketcheson, 1973). Les interactions entre les conditions de milieu n'étant pas prises en compte, les résultats obtenus ne sont pas généralisables à des situations autres que celles reproduisant les conditions expérimentales, et ne peuvent donc être utilisées pour décrire précisément le développement du système racinaire.

II existe par ailleurs un certain nombre de modèles théoriques d'architecture (Lungley, 1973; Diggle, 1988; Pages et Aries, 1988), qui n'ont jamais été paramétrés. Afin d'adapter ces modèles à différentes situations pédo-climatiques, ceci devra se faire en privilégiant les facteurs qui confèrent une certaine stabilité à l'expression de la structure : synchronisme entre organogenèse aérienne et racinaire, croissance allométrique de ces 2 sous-systèmes, influence du génotype sur les caractères morphologiques...

C'est ce travail que nous nous proposons d'effectuer pour le maïs (variété Dea). Les rythmes d'émission des racines primaires (terminologie définie par Girardin et al, 1986) étant déjà connus, (Picard et al, 1985) cette étude portera principalement sur la ramification secondaire et aura pour but :

- d'une part, de définir la structure d'un réseau racinaire, c'est-à-dire, (i) de caractériser chaque partie d'une racine d'ordre 1 (ou racine primaire) en fonction de son aptitude à porter des ramifications, et (ii), de déterminer le nombre et le devenir de ces racines d'ordre 2 (encore appelées racines secondaires);

- d'autre part, de suivre la cinétique d'émission des secondaires le long des primaires.

Cependant, les techniques classiques d'étude des enracinements (Bohm, 1979) ne nous permettent pas de mesurer tous ces paramètres à l'échelle qui nous intéresse : celle de la parcelle cultivée. Des expérimentations en rhizotrons, (méthode qui a fait l'objet d'une évaluation préliminaire, Jordan, 1991a) ont donc été menées en parallèle avec les observations au champ : prélèvement de plantes et de monolithes de terre.

\section{DISPOSITIF EXPÉRIMENTAL}

Les essais effectués de 1983 à 1985 ont déjà été décrits de façon détaillée (Jordan, 1986). Seuls les points indispensables à la compréhension des résultats seront repris ici.

\section{Le dispositif}

\section{Les rhizotrons}

Six rhizotrons sont utilisés en 1984 et 12 en 1985 , où l'utilisation d'une plaque de sélection des primaires, décrite ultérieurement, limite le nombre d'observations.

La section des rhizotrons est de $80 \times 20 \mathrm{~cm}$. La profondeur est de $1 \mathrm{~m}$, Grimes et al (1975) 
ayant montré qu'en conditions irriguées, $99 \%$ de la matière sèche racinaire était située dans les 90 premiers $\mathrm{cm}$ de sol.

Le caisson est en PVC et isolé thermiquement à l'aide de polystyrène expansé. Il est incliné de $15^{\circ}$ du côté de la paroi transparente en plexiglas. Entre 2 mesures, celle-ci est recouverte d'un cache de PVC opaque.

En 1984, nous avons été gênés dans nos observations par des chevauchements consécutifs à la présence d'un nombre d'organes trop important au contact du plexiglas. En 1985, 8 rhizotrons ont donc été équipés d'une plaque de PVC écartant le système racinaire de cette paroi (Jordan, 1991a). Seules 4 racines, choisies en fonction du rang de leur entre-nœud d'insertion, sont amenées au contact de la paroi : pour cela elles sont passées au travers de trous percés dans la plaque, prolongés par une gaine de PVC jusqu'à la paroi.

Les caissons sont placés sous abri mobile et remplis avec un sol remanié dont les caractéristiques physico-chimiques sont celles du sol de l'une des parcelles expérimentales, celle de Colmar 1. Le profil du sol a été reconstitué horizon par horizon en respectant les densités apparentes. Une couche drainante d'une épaisseur de $10 \mathrm{~cm}$, composée de gravier et de sable, occupe le fond des rhizotrons. Le substrat présente donc une hétérogénéité verticale comme cela se rencontre fréquemment à l'échelle de la parcelle cultivée.

Les semis, de 4 graines par caisson, sont effectués le 9 mai en 1984 et le 24 avril en 1985. Au stade 3 feuilles, un seul pied, choisi de façon à ce que la population soit la plus homogène possible, est conservé. Le décalage des dates de semis se retrouve à la floraison femelle qui a lieu le 10 août en 1984 et le 30 juillet en 1985.

\section{Le champ}

L'expérimentation a été réalisée en 1983 et 1984, sur 6 parcelles différenciées du point de vue des itinéraires techniques et des localisations géographiques, afin de permettre l'expression de la variabilité phénotypique du processus de ramification.

Les sites ont été choisis de façon à inclure une certaine variabilité climatique, notamment du point de vue de la pluviométrie totale qui, pour les mois d'avril à octobre, varie de 347 à $623 \mathrm{~mm}$ entre les différentes localisations.

Les sols des stations sont formés sur loess et présentent tous une texture de limon argileux avec une teneur en matière organique faible à modérée, un $\mathrm{pH}$ alcalin ou légèrement acide.

Les techniques culturales ont été laissées à l'initiative des agriculteurs. Notons que, sur la parcelle de Flaxlanden-1983, un traitement accidentel à base d'hormones a considérablement réduit l'organogenèse sur l'ensemble des entrenœuds, sans pour autant pénaliser la production de biomasse aérienne.

Les semis, d'une densité comprise entre 7,4 et $9,5 \mathrm{pieds} / \mathrm{m}^{2}$ ont été échelonnés du 24 avril au 29 avril en 1983 et du 23 avril au 3 mai en 1984; les floraisons femelles du 18 juillet au 25 juillet en 1983 et du 30 juillet au 8 août en 1984 . Les rendements en grains ne varient pas d'une année à l'autre et sont compris entre 78 et 103 q/ha.

\section{Les mesures}

\section{Paramètres caractérisant la ramification secondaire}

Chaque racine primaire est composée de 3 parties distinctes (fig 1) : 2 zones non ramifiées $a$ et $b$ situées respectivement en-deçà de l'apex et à la base, au-delà du point de raccordement à la tige, entourent la partie centrale $r$, d'où sont issues les secondaires.

Nos mesures ont pour objet de déterminer les longueurs de $a$ et de $b$, les nombres de secondaires par $\mathrm{cm}$ de primaire et de suivre l'évolution de ces paramètres au cours du cycle cultural. Dans cette étude, nous ne tiendrons pas compte des primaires issues des entre-ncuds de rang supérieur ou égal à 8 , qui pour la variété Dea sont en général émises au-dessus du niveau du sol.

\section{Mesures}

Chaque fois que cela est possible, les évaluations sont faites au champ. Cependant, les rhizotrons sont indispensables, d'une part, pour effectuer des mesures au-delà de $35 \mathrm{~cm}$ de profondeur et, d'autre part, pour suivre la cinétique de ramification.

Au champ, l'essentiel des observations a été effectué dans la couche labourée (horizon 0/35 $\mathrm{cm}$ ) à partir de prélèvements de :

- 20 plantes déterrées chaque semaine à la bêche sur l'une des parcelles, celle de Colmar 1 . Pour chaque primaire, $b$ est mesurée à l'aide 


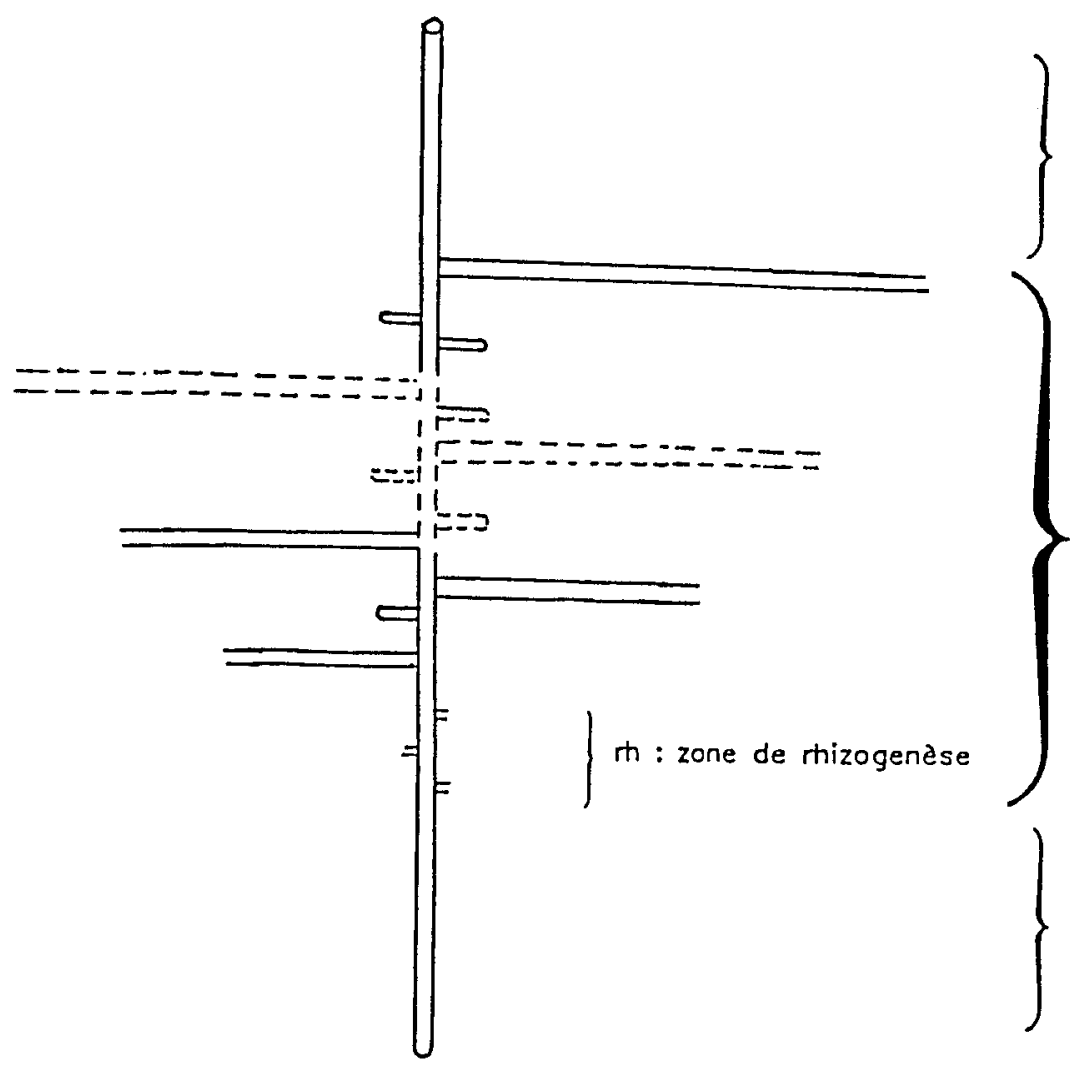

b : portie non ramifiée situes a la bose de la racine sous son point de roccoridement

$r$ : zone ramifié dont lo longueur s'accroit pendont la croissonce

o : partie non ramifiée située en-deça de l'extrémité apicale

Fig 1. Différentes parties d'une racine.

d'une réglette métallique. Afin de pouvoir évaluer les données obtenues en rhizotrons, et bien qu'il soit extrêmement difficile de prélever des racines entières, la longueur de a sur les primaires issues de l'entre-nœud 2 est également mesurée pour l'une des dates.

- 2 planches de fakir, d'une largeur de $82 \mathrm{~cm}$, d'une profondeur de $42 \mathrm{~cm}$ et d'une épaisseur de $15 \mathrm{~cm}$. Pour les 6 sites, les prélèvements ont eu lieu à la floraison, selon un procédé décrit par De Roo (1957), Nelson et Allmaras (1969), les plantes échantillonnées se trouvant au centre de la section $82 \times 15 \mathrm{~cm}$ du monolithe de terre.

La terre est éliminée à l'aide d'un jet d'eau à faible pression. Puis, pour chaque primaire, le nombre de ramifications est évalué par tronçons de $5 \mathrm{~cm}$ à partir du point d'insertion sur la tige. Les comptages se font à la binoculaire, en raison du nombre important de secondaires et de la nécessité de comptabiliser les moignons des organes brisés au cours des manipulations. Compte tenu de l'état du matériel végétal, il n'a pas été possibie de regrouper les racines d'ordre 2 en classes de longueur.

En rhizotrons, chaque primaire au contact de la vitre est individualisée et le numéro de l'entre- nceud dont elle est issue déduit du phyllochrone (d'après les résultats de Picard et al, 1985). Sa croissance est relevée journellement à l'aide d'un calque. Par ailleurs, 2 fois par semaine, $a$ est mesuré et les secondaires sont dénombrées et regroupées en fonction de leur longueur (inférieure ou supérieure à $3 \mathrm{~cm}$ ). Ces évaluations se font également par tronçon de $5 \mathrm{~cm}$.

À la récolte, la paroi transparente ainsi que les primaires au contact de celle-ci sont otées. Le système racinaire restant, pris dans la motte de terre, est dégagé par aspersion, et les mêmes comptages sont à nouveau réalisés à la binoculaire sur chaque primaire.

\section{RÉSULTATS}

Les déterminations ayant eu lieu sur toutes les primaires disponibles, le nombre de répétitions par type de données est très variable (tableau I).

Les résultats ont principalement été traités à l'aide d'analyses de variance en modèle linéaire général et de tests de Bonferroni (comparaison de moyennes). 
Tableau I. Nombres de répétitions par type de mesure.

\begin{tabular}{lllllllllllll}
\hline Méthode & Horizon & $E_{2}$ & $E_{3}$ & $E_{4}$ & $E_{5}$ & $E_{6}$ & $E_{7}$ \\
\hline
\end{tabular}

\section{Mesures de a}

Rhizotrons : 1984

Rhizotrons : 1985

Prélèvements de plantes : 1984

Rhizotrons : 1984

Prélèvements de plantes : 1984

Planches de fakir : 1883 et 1984

$\begin{array}{rrrrrr}63 & 24 & 23 & 14 & 22 & 24 \\ 1 & 8 & 6 & 3 & 4 & 5 \\ 28 & & & & \end{array}$

\section{Mesures de b}

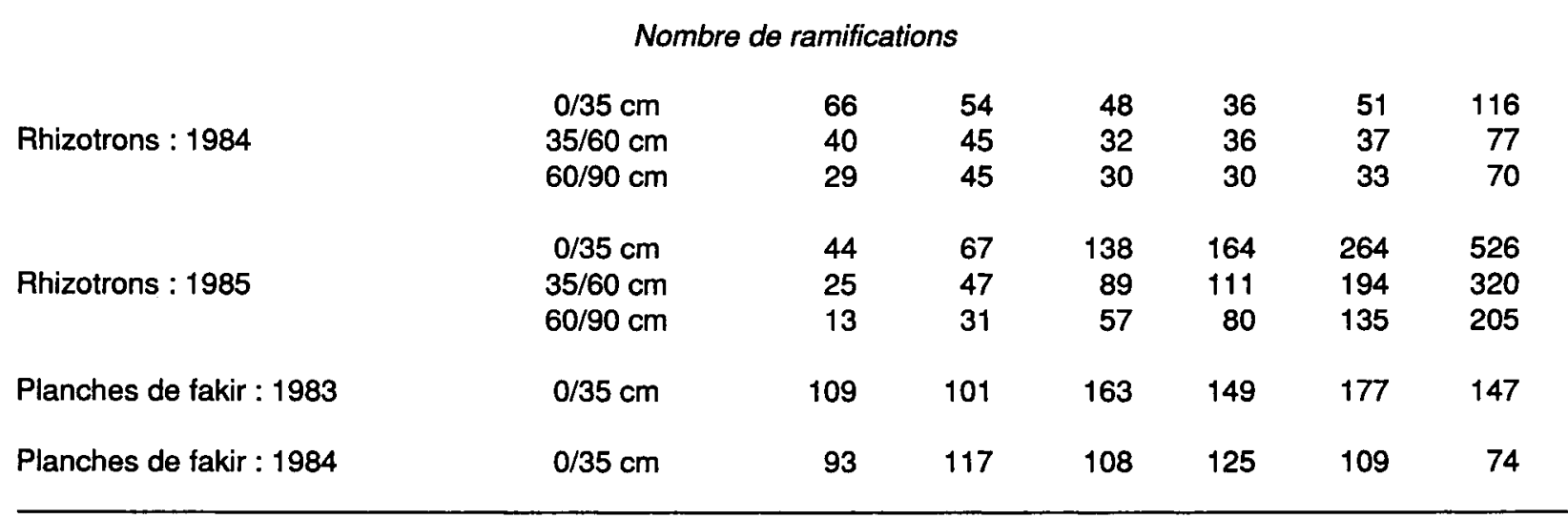

\section{La structure du réseau}

\section{Portions de primaires non ramifiées : $\boldsymbol{a}$ et $\boldsymbol{b}$}

On connaît de longue date la présence de a (Lungley, 1973) et le mécanisme de régulation hormonale qui inhibe l'organogenèse sur cette portion de racine qui correspond aux zones de différentiation et d'élongation de la primaire (Torrey, 1976; Wightman et al, 1980). Mais, à notre connaissance, aucun travail n'a jamais fait mention de $b$.

Les longueurs de a (fig 2) sont comprises entre 10,3 et $19,3 \mathrm{~cm}$ en 1984 et entre 12 et 19,1 $\mathrm{cm}$ en 1985. Pour $b$, elles varient de 0,40 aे 3,42 $\mathrm{cm}$ en 1984, et de 0,37 à 3,62 cm en 1985 .

Des tests de comparaisons des valeurs moyennes, effectués entre-nœud par entrenœud au seuil $5 \%$ montrent :

- l'homogénéité des résultats, obtenus par les différentes méthodes concernant $b$. Pour $a$, il semblerait, au vu des mesures effectuées sur l'entre- nœud 2, que les résultats rhizotrons ne soient pas extrapolables aux conditions du champ;

- peu d'influence des conditions pédoclimatiques. En effet, sauf pour les valeurs de a concernant $E_{7}$, les différences entre les longueurs moyennes évaluées (cas 1) pour chacune des 2 années, et (cas 2), des 6 localisations (résultats "planches de fakir", portant uniquement sur $b$ ne sont pas significatives;

- la stabilité, sur l'ensemble du cycle de culture, des longueurs de a (résultats obtenus en rhizotrons) et de $b$ (évaluations à partir des prélèvements de plantes au champ). Ceci tend à montrer que l'émission racinaire est limitée à la zone $r$ (fig 1) et que chaque primaire se ramifie dès qu'elle atteint une longueur seuil déterminée par le génotype et égale à $(a+b)$.

Les valeurs de a ne dépendent pas du rang de l'entre-nœud d'insertion de la racine primaire sur la tige (noté $E_{i}$ ), mais la longueur de $b$ peut être déduite de $E_{i}$ par la relation suivante (calculée à partir des prélèvements de plantes) : 
Légende: 0 "a";* "b"

Rhizotrons 1984
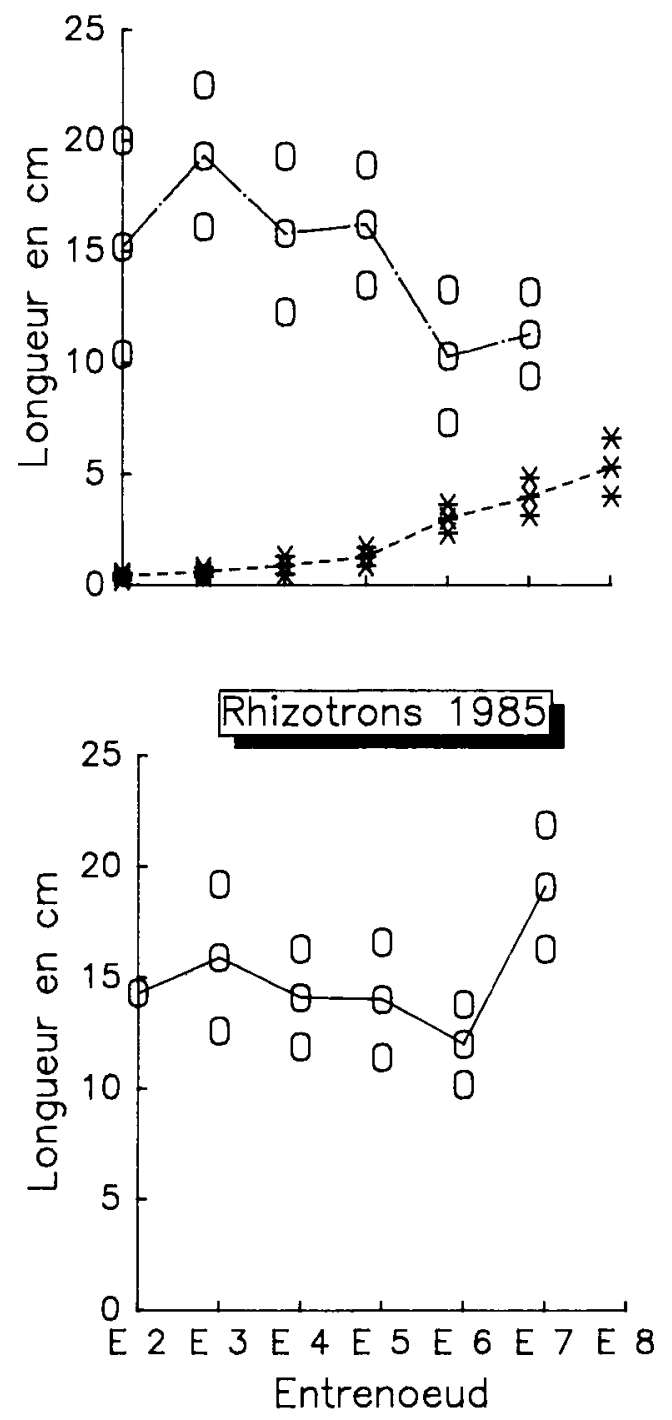

Planches de Fakir 1983 et 1984
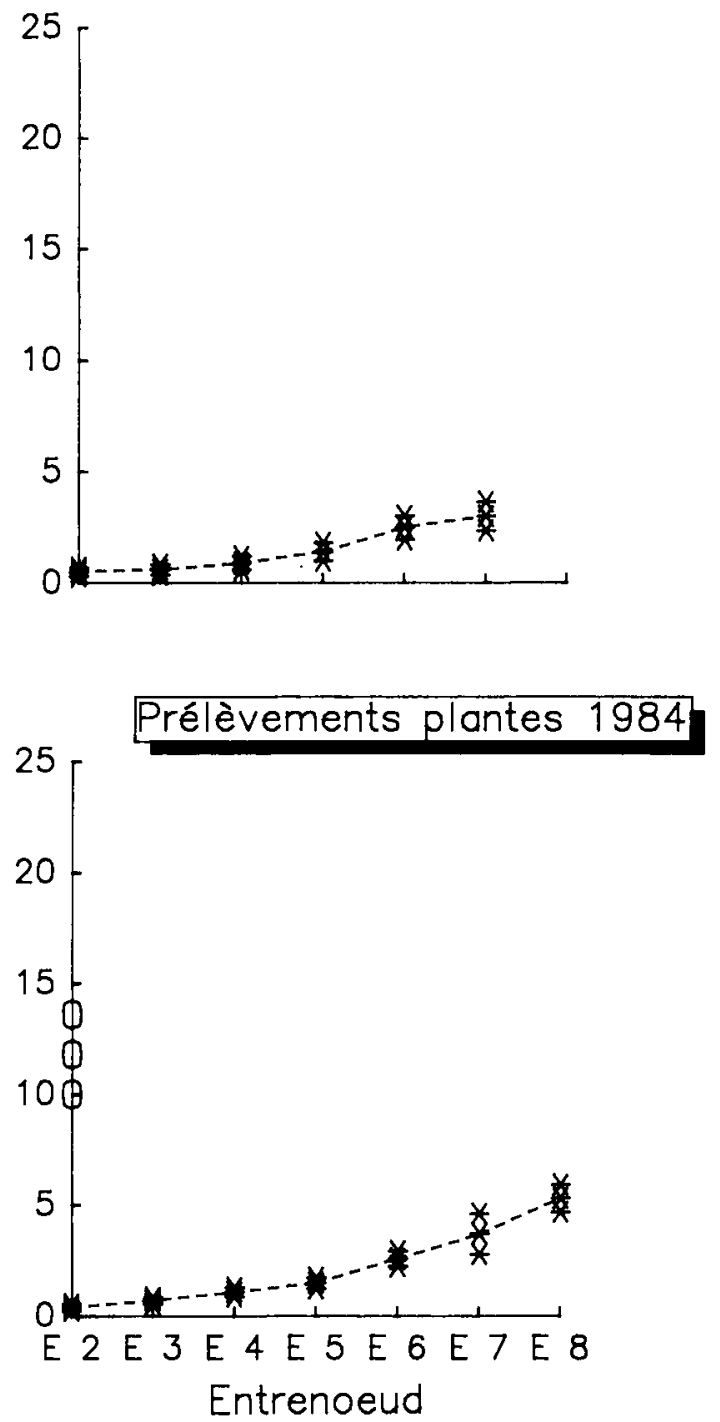

Fig 2. Longueur des parties non ramifiées $a$ et $b$ (fig 1) en fonction de l'entre-nœud d'insertion de la racine primaire pour les 2 années et les différentes méthodes de mesure.

$$
\operatorname{Ln} b=1,85 \operatorname{Ln} E_{i}-2,33 ; r=0,99^{\star \star \star} ; 1075 \mathrm{dl}
$$

Le coefficient de la régression baisse légèrement, mais de façon non significative (test de Student, seuil 1\%) s'il est établi à partir des résultats des planches de fakir. La relation devient :

$$
\operatorname{Ln} b=1,64 \operatorname{Ln} E_{i}-2,03 ; r=0,97^{\star \star \star} ; 522 \mathrm{dl}
$$

La précision de cette estimation est en partie due au fait que les coefficients de variation sur $b$ sont relativement faibles : les $\mathrm{CV}$ (évalués à partir des plantes prélevées au champ) sont compris entre 14 et $27 \%$ sauf pour l'entre-nœud 7 où la

*** $: P \leq 0,1 \%$. 
valeur (égale à $87 \%$ ) est inexplicablement élevée.

Sur a, les CV sont toujours supérieurs à $100 \%$, ce qui, dans les tests de comparaison de moyennes décrits précédemment a pu masquer l'influence du pédo-climat et du stade de développement. Cette dispersion n'est pas liée au fait que l'on travaille sur plusieurs plantes. En effet, les écarts entre les observations sur les racines issues d'un même pied sont du même ordre de grandeur, vraisemblablement parce que toutes les plantes ont le même génome, Dea étant un hybride simple.

\section{Nombre de racines secondaires totales}

Les résultats du champ sont présentés figure 3 en fonction de l'année et de l'entre-nœud d'insertion de la primaire, une analyse de variance (tableau II) ayant montré que ces 2 facteurs sont significatifs au seuil $5 \%$.

Le nombre de racines d'ordre 2 par $\mathrm{cm}$ de primaire varie de 6,5 à 13,1 en 1983 et de 6,1 à 16 en 1984. II augmente avec le rang de l'entrenœud. Un test de comparaison de moyennes montre que, pour les 2 années d'étude, les racines d'ordre 1 issues de $E_{2}$ ont significativement moins de secondaires que celles portées par les entre-nœuds intermédiaires, elles-mêmes moins ramifiées que les réseaux insérés sur $E_{7}$.
L'importance des écarts interannuels atteste que le processus d'émission des racines d'ordre 2 dépend du milieu. Cependant, il n'a pas été possible de mettre en évidence de différences entre les 6 sites d'étude. Ceux-ci ont pourtant été choisis de façon à intégrer le transect climatique Nord/Sud de la plaine d'Alsace. Les différences entre les 2 années ne sont certainement pas liées à la température, car en 1984, où les moyennes mensuelles sont inférieures à celles de 1983 d'environ $2{ }^{\circ} \mathrm{C}$, le nombre de ramifications est plus élevé pour l'ensemble des entrenœuds sauf $E_{2}$, alors que normalement ces 2 facteurs sont corrélés positivement (Cooper, 1973).

L'interaction site-année est significative, en raison de l'accident cultural intervenu sur la parcelle de Flaxlanden-1983.

Les coefficients de variations sur ces données sont compris entre 23 et $35 \%$. Ils sont du même ordre de grandeur les 2 années d'étude et expriment la variabilité inhérente au processus d'émission des secondaires (Jordan, 1991a).

Les observations en rhizotrons sur les organes au cœur du monolithe de terre (fig 4), nous permettent de compléter ces résultats en évaluant leur constance avec la profondeur. Deux modes d'expression de ce paramètre sont utilisés (tableau II) :

- (i) l'un relatif au milieu (analyse $x$ ) : discrimination en 3 classes correspondant aux portions de

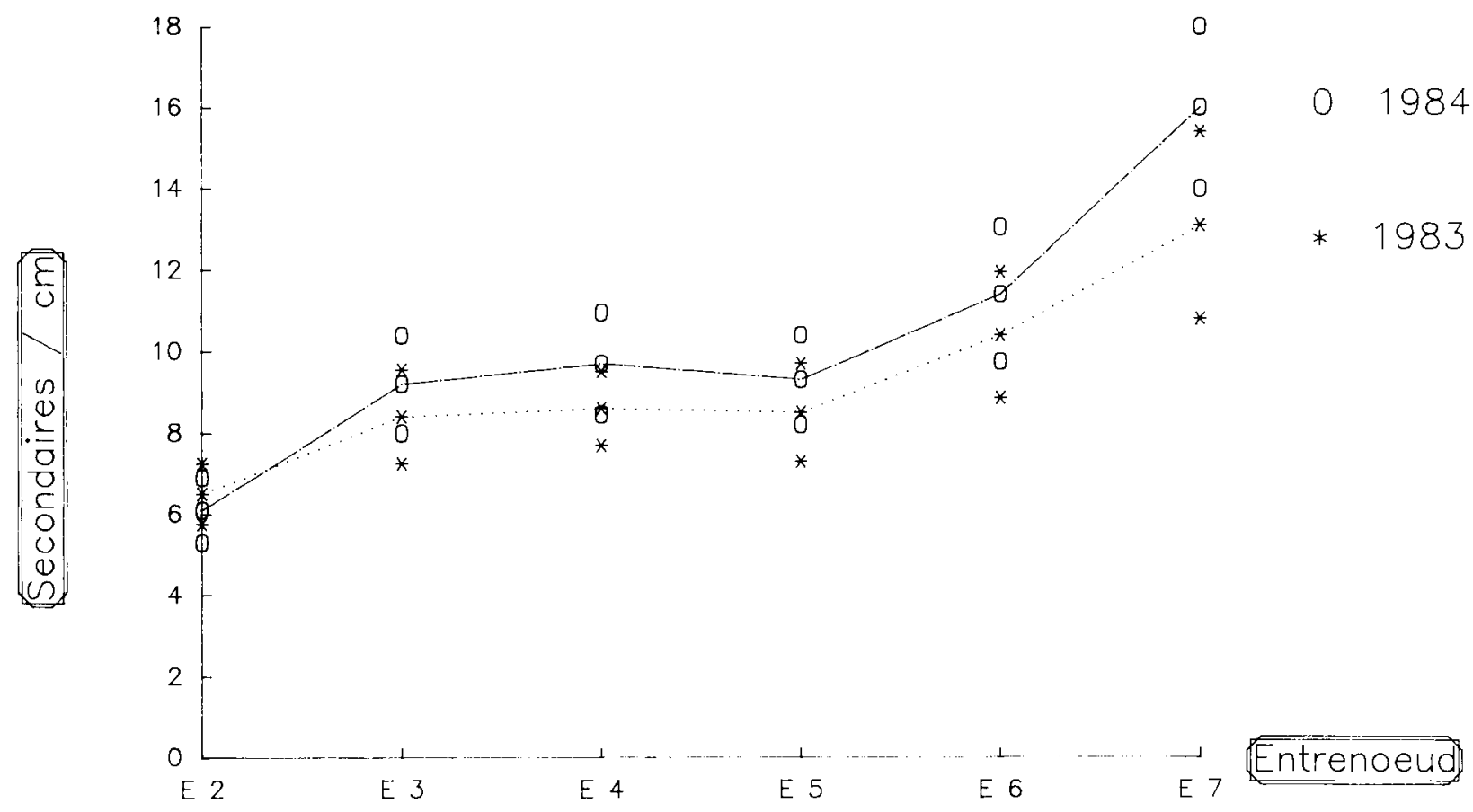

Fig 3. Moyenne du nombre total de secondaires par $\mathrm{cm}$ en fonction du rang d'insertion de la racine primaire. Résultats 1983 et 1984 obtenus à l'aide des planches de fakir pour l'horizon $0 / 35 \mathrm{~cm}$. 
Tableau II. Résultats des analyses de variance.

\begin{tabular}{|c|c|c|c|c|c|}
\hline \multirow{3}{*}{ Facteur } & \multirow{2}{*}{\multicolumn{2}{|c|}{$D D L$}} & \multirow{2}{*}{\multicolumn{3}{|c|}{ Analyse }} \\
\hline & & & & & \\
\hline & $(x-y)$ & (z) & $(x)$ & $(y)$ & $(z)$ \\
\hline \multicolumn{6}{|l|}{ Rhizotrons* } \\
\hline $\begin{array}{l}\text { Année } \\
\text { Profondeur }(35 / 90 \mathrm{~cm}) \\
\text { Entre-nœud } \\
\text { Année * Entre-nœud } \\
\text { Année * Profondeur } \\
\text { Entre-nœud * Profondeur } \\
\text { Résiduelle }\end{array}$ & $\begin{array}{r}1 \\
2 \\
5 \\
5 \\
2 \\
10 \\
10\end{array}$ & $\begin{array}{r}(1) \\
(8) \\
(5) \\
(5) \\
(8) \\
(32) \\
(32)\end{array}$ & $\begin{array}{c}0,57^{\star \star} \\
30,89^{\star \star} \\
20,21^{\star \star} \\
3,74 \\
1,73 \\
1,13\end{array}$ & $\begin{array}{l}\left(30,07^{\star \star}\right) \\
(1,71) \\
\left(4,19^{\star \star}\right) \\
(4,15) \\
\left(3,38^{\star \star}\right) \\
(1,65)\end{array}$ & $\begin{array}{c}11,46^{\star *} \\
4,44 \\
24,36^{\star \star} \\
4,54 \\
0,33 \\
2,04\end{array}$ \\
\hline \multicolumn{6}{|l|}{ Planches de Fakir } \\
\hline $\begin{array}{l}\text { Site } \\
\text { Année } \\
\text { Entre-nœud } \\
\text { Site * Année } \\
\text { Site * Entre-nœud } \\
\text { Année * Entre-nœud } \\
\text { Résiduelle }\end{array}$ & $\begin{array}{r}54 \\
1 \\
5 \\
5 \\
24 \\
5 \\
26\end{array}$ & & & $\begin{array}{c}2,62 \\
7,26^{\star \star} \\
26,22^{\star *} \\
3,03^{\star *} \\
1,38 \\
0,77\end{array}$ & \\
\hline
\end{tabular}

- Analyses $x$ et $y$ : nombre total de secondaires en fonction de 2 modes d'expression de la profondeur : $(x)$ : discrimination par horizon de sol $(0 / 35,35 / 60$ et $60 / 90 \mathrm{~cm})$. (y) : discrimination en fonction des 9 portions de $5 \mathrm{~cm}$ de primaires sur lesquelles ont été fait les comptages. Analyse z : nombre de secondaires allongées, profondeur donnée en fonction des 3 horizons de sol.

racines d'ordre 1 colonisant chacun des 3 horizons de sol $(0-35 \mathrm{~cm}, 35-60 \mathrm{~cm}$ et $60-90 \mathrm{~cm})$. Dans ce cas, l'effet profondeur est significatif au seuil $5 \%$;

- (ii) l'autre, plus spécifique de la morphologie racinaire (analyse $y$ ) : distance entre chacun des 9 tronçons de $5 \mathrm{~cm}$ sur lesquels ont été faits les comptages et le point d'insertion de la primaire sur la tige. Dans ce cas, l'influence du facteur étudié s'exprime uniquement au travers de l'interaction année-profondeur.

D'une manière générale, le nombre de secondaires par unité de longueur diminue avec la profondeur, soit pour des raisons physiologiques, soit parce que le profil de sol est constitué de 3 horizons bien différenciés. Mais, si la classification est insuffisamment discriminante (cas ii), cette tendance est masquée par la variabilité inhérente au processus de ramification.

\section{Nombre de racines secondaires allongées}

Les secondaires une fois émises, vont soit s'allonger et se ramifier, soit, arrêter toute croissance et garder une longueur inférieure à $3 \mathrm{~cm}$.
Dans les 2 cas, les durées de vie (estimées à l'œil par changement de coloration) sont sensiblement égales à celle de la plante.

Les racines longues ont été quantifiées en rhizotrons, sur les primaires au cœur du monolithe de terre (fig 4). Les formes des courbes décrivant l'évolution de leur nombre en fonction du rang d'insertion de la racine primaire sont identiques pour les 2 années et les 3 horizons pris en compte. En effet, une analyse de variance (tableau II) montre qu'il n'y a pas d'interaction entre ces facteurs. Le plus souvent, les courbes sont ascendantes jusqu'à $E_{5}$ puis décroissantes.

Aucune baisse sensible n'est observée avec la profondeur, contrairement à ce qui se passe pour le nombre total de ramifications. En conséquence, la proportion de secondaires qui s'allonge augmente avec la distance à la base de la racine primaire. C'est ainsi que sur $E_{5}-1984$ le pourcentage de racines longues passe respectivement de 21 à 32 puis $40 \%$ entre les horizons $0 / 35,35 / 60$ et $60 / 90 \mathrm{~cm}$.

L'influence de l'année est double. Elle porte à la fois :

\footnotetext{
$*{ }^{* *} \mathrm{P} \leq 5 \%$.
} 


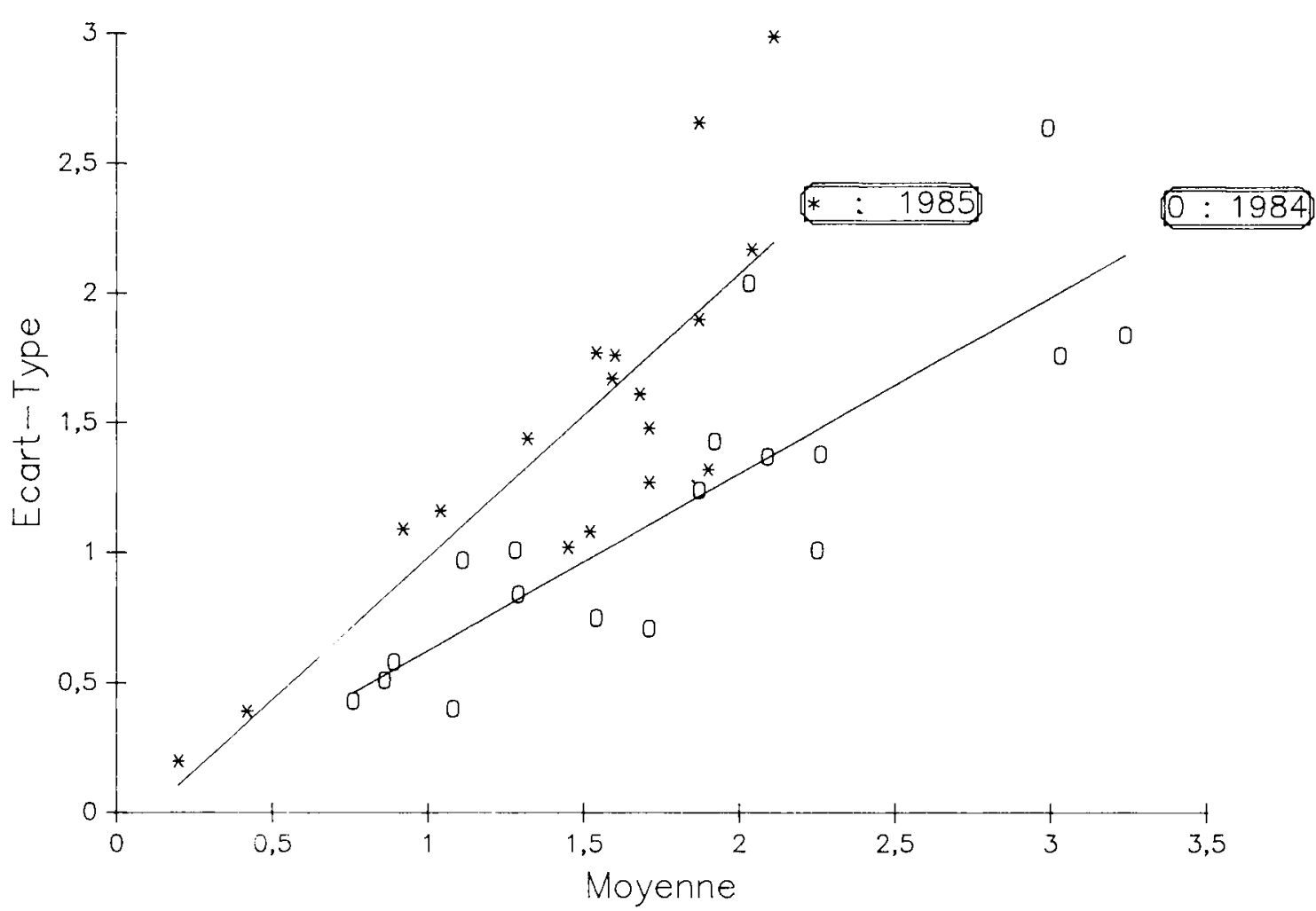

Flg 4. Valeurs des écarts types en fonction des valeurs moyennes des nombres de secondaires allongées pour les 2 années d'átude.

- sur les valeurs moyennes. Le tableau II montre que l'effet de ce facteur est significatif au seuil $5 \%$. Les résultats obtenus en 1984 sont supérieurs de 3 à $13 \%$ à ceux de 1985;

- sur la variabilité des comptages. Une relation différente, spécifique à chacune des 2 années (fig 5) permet d'estimer les écarts types $(E-T)$ à partir des moyennes $(X)$ :

Pour $1984: E-T=0,68 X+0,20 ; r=0,85^{\star \star *} ; 17 \mathrm{dl}$ Pour 1985 : $E-T=1,09 X-0,11 ; r=0,83^{* * *} ; 17 \mathrm{dl}$

Les CV qui en découlent sont compris entre 37 et $100 \%$ en 1984 et entre 69 et $142 \%$ en 1985 . Ils sont donc beaucoup plus élevés que ceux établis pour les ramifications totales, ce qui confirme la prédominance des facteurs du milieu sur l'allongement des secondaires.

\section{La cinétique d'émission}

Les résultats présentés sont ceux obtenus en rhizotrons, à partir des observations effectuées à travers la paroi transparente pendant toute la durée du cycle de culture.

\section{Localisation de la zone de ramification}

Une primaire se ramifie dès que sa longueur est supérieure à $(a+b)$. Au fur et à mesure de sa croissance, des secondaires sont émises dans la zone de rhizogenèse "rh" qui correspond aux 5 derniers $\mathrm{cm}$ environ de la partie apicale de «r» (fig 1). En effet, nous avons montré que :

- les longueurs de $a$ et de $b$ ne varient pas au cours du cycle (paragraphe 1);

- pour toutes les portions de racines primaires (tronçons de $5 \mathrm{~cm}$ ) prises en compte au moment des mesures, le nombre de ramifications devient strictement constant en fonction du temps, au plus tard, 12 j après l'apparition des premières secondaires.

Cette durée est plus importante en début qu'en fin de cycle et passe d'une semaine et demie (intervalle séparant 4 comptages) à une semaine, parfois à $4 \mathrm{j}$. Ceci découle du fait que les vitesses d'élongation racinaire sont plus élevées en période estivale (Jordan, 1986), ce qui a pour conséquence de réduire le temps imparti à la ramification d'une portion de primaire. 
$19735 \mathrm{~cm}$

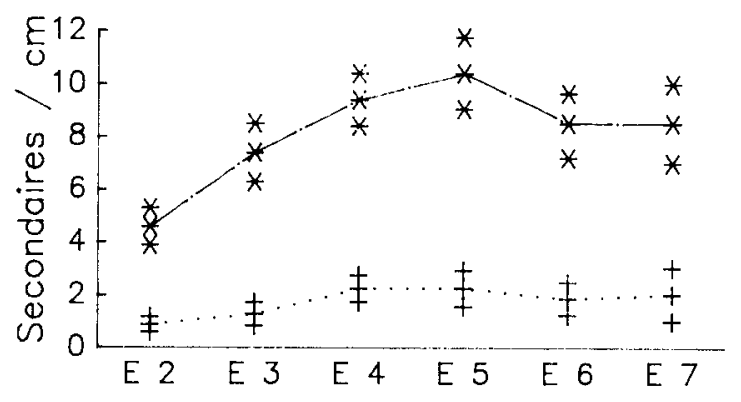

$35 / 60 \mathrm{~cm}$

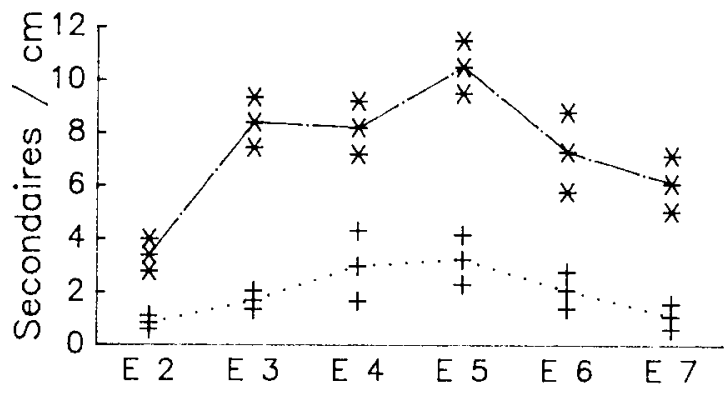

\section{$60 / 90 \mathrm{~cm}$}

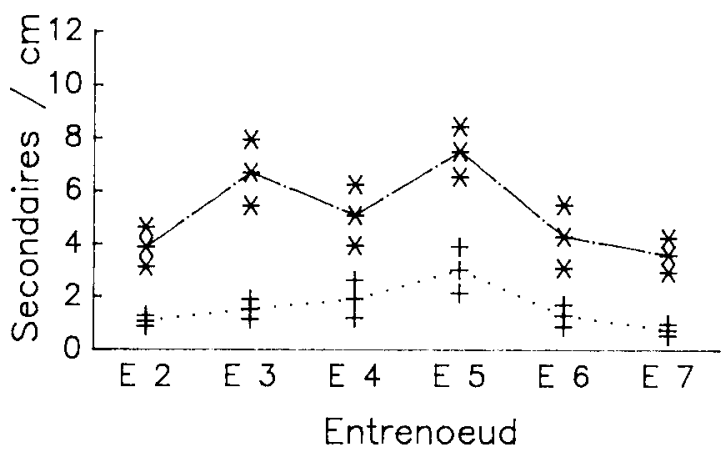

*: Rocines totales, + Racines allongées

\section{5}
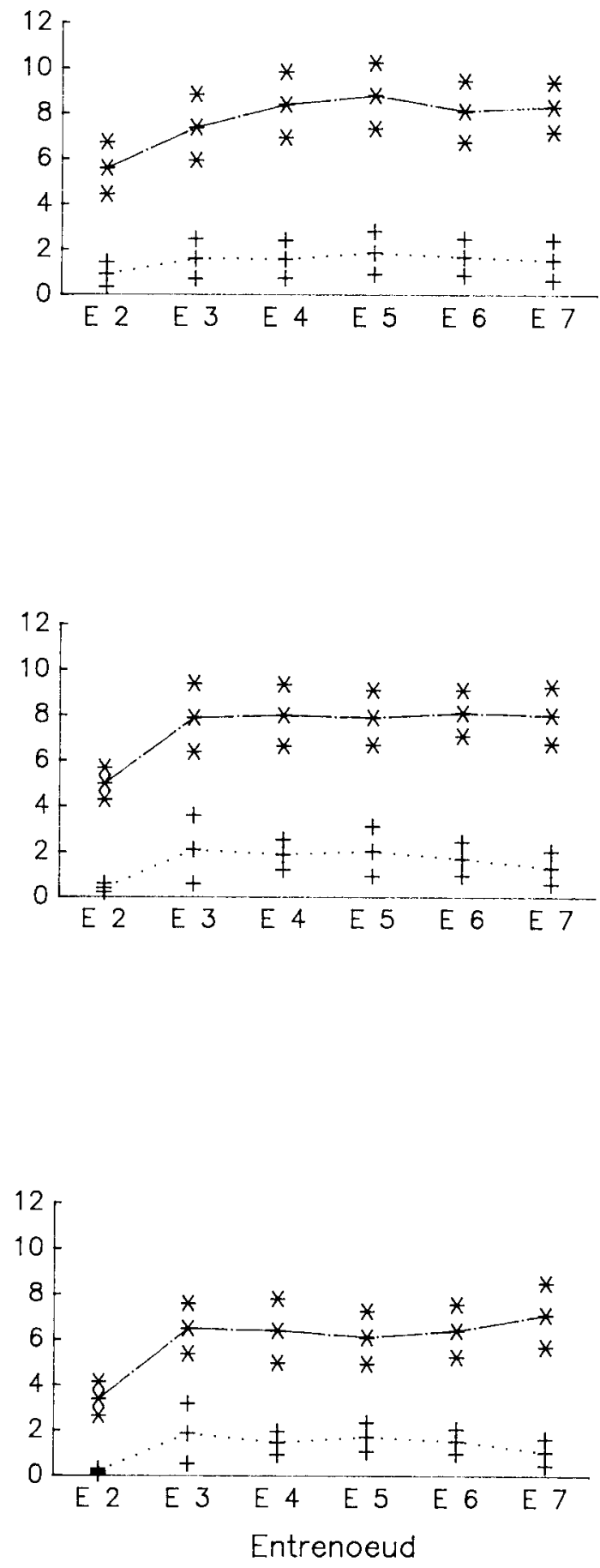

Fig 5. Nombre moyen de secondaires totales et de secondaires allongées par $\mathrm{cm}$ en fonction du rang d'insertion de la racine primaire. Résultats 1984 et 1985 obtenus en rhizotrons sur les primaires au cœur du monolithe de terre pour les horizons 0/35, 35/60 et $60 / 90 \mathrm{~cm}$. 


\section{Ramification des primaires émises} sur les différents entre-nœuds

Les figures $6 a$ et $6 b$ permettent de localiser, pour chacun des entre-nœuds, la zone de rhizogenèse "rh" (profondeur à laquelle sont émises les ramifications) en fonction du nombre de jours après semis et du phyllochrone (défini par Gounot et al, 1980). L'intervalle entre les comptages étant de 3 ou 4 jours, l'incertitude sur les résultats est de cet ordre de grandeur. En 1985, les mesures ont débuté à $35 \mathrm{~cm}$ de profondeur et concernent uniquement les entre-nœuds de rang supérieur à 2 en raison de la présence, sur les rhizotrons, de la plaque de sélection des primaires.

Ce dispositif a provoqué un arrêt de croissance au moment du passage des premières racines de l'horizon de surface composé d'argile expansée aux horizons profonds (limon argileux). Ceci a certainement contribué au décalage interannuel observé sur les cinétiques de ramification exprimés en fonction du nombre de jours après semis. Mais, l'utilisation du phyllochrone comme norme de temps permet de les regrouper sur une courbe unique comme cela est le cas pour les rythmes d'émission des racines primaires (Picard et al, 1985). Notons la présence physiologiquement inexpliquée, en 1984, d'arrêts et de ruptures de pentes visibles aux mêmes dates pour les entre-nœuds de rang inférieur ou égal à 3. Ces changements ne peuvent pas être reliés au début de l'émission sur les entre-nœuds supérieurs mais correspondent à un arrêt de la ramification entre 6,5 et 7,5 feuilles d'une part et 9,8 et 11 feuilles, d'autre part.

L'émission secondaire est liée à un double déterminisme :

- le début de la ramification sur une primaire insérée à l'entre-nœud $E_{i}$ dépend du phyllochrone. Cette dépendance entre organogenèse aérienne et racinaire est encore vérifiée au moment où la portion de primaire visible à travers la vitre du rhizotron, commence à se ramifier (émission de secondaires à environ $12 \mathrm{~cm}$ de la graine). En effet, cette date, qui n'est pas celle du début de l'émission secondaire pour l'entre-nœud $E_{i}$ (longueurs de $b$ comprises entre 0,4 et $4 \mathrm{~cm}$ ) peut être déduite du nombre de feuilles visibles (NF) par la relation :

$$
N F=2,20 E_{i}+0,91 ; r=0,99^{\star \star \star} ; 5 \text { dl pour } i<7
$$

Ce calcul n'est possible qu'en 1984, mais il est fortement probable (fig 6) que les différences interannuelles sont négligeables.
La progression de la zone de rhizogenèse «rh» le long d'une racine d'ordre 1 est proportionnelle à la vitesse de croissance de cette dernière. Quand les primaires sont entièrement ramifiées sur leurs 90 premiers $\mathrm{cm}$ (hauteur utile du rhizotron) il n'y a plus aucune concordance entre $E_{i}$ et NF (tableau III) car la durée en temps phyllochronique nécessaire à l'émission de la totalité des secondaires en-deçà de cette profondeur de $90 \mathrm{~cm}$ varie fortement, et de façon non linéaire, avec le rang de $E_{i}$. Elle est maximale, égale à 8,3 pour $E_{2}$, baisse jusqu'à 3,8 pour $E_{3}$ et $E_{4}$, et enfin réaugmente de façon non significative (valeur de 4,1 ) pour $E_{5}$. Les primaires issues des entre-nœuds de rang supérieurs à 5 s'allongent, donc se ramifient, après la fin de l'émission foliaire.

\section{DISCUSSION ET CONCLUSION}

Ce travail nous a permis de quantifier les paramètres relatifs à la ramification des racines nodales primaires du maïs, indispensables à l'élaboration d'un modèle de structure représentatif des conditions réelles de culture.

Les différents résultats obtenus seront discutés point par point.

\section{Les parties non ramifiées}

Nos données peuvent, dès à présent, être utilisées pour paramétrer les modèles d'architecture existants (Diggle, 1988; Pagès et Aries, 1988) car ils présentent, du moins pour $b$, une certaine stabilité en fonction de l'année et du lieu.

L'importance des variations locales des conditions de milieu sur les valeurs de a est attestée par la variabilité inter- et intra-plante. Le rôle de l'état hydrique du sol semble prépondérant (Lambers et Posthumus, 1980).

Les longueurs de $b$ sont plus stables et augmentent avec le rang de l'entre-nœud. Cela est généralement le cas des paramètres descriptifs de la structure racinaire : diamètres et nombres des racines d'ordre 1, (Jordan, 1986), et, dans une moindre mesure, de celles d'ordre 2 (résultats non publiés en totalité).

\section{Les nombres de racines secondaires totales et allongées}

Nos observations vont dans le même sens que celles effectuées sur maïs par Yamazaki et Kae- 
a

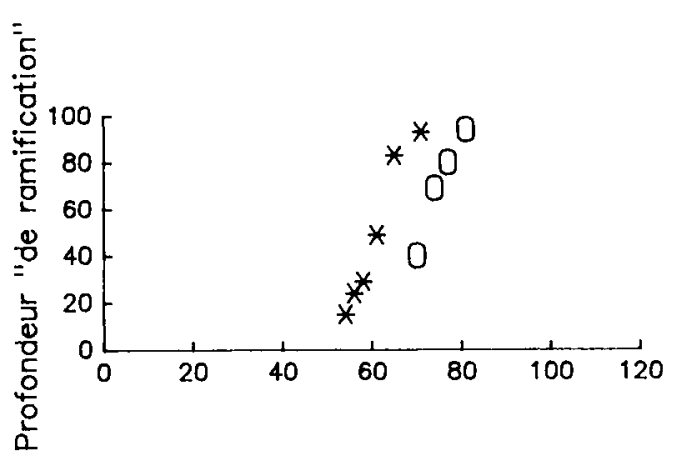

E 4
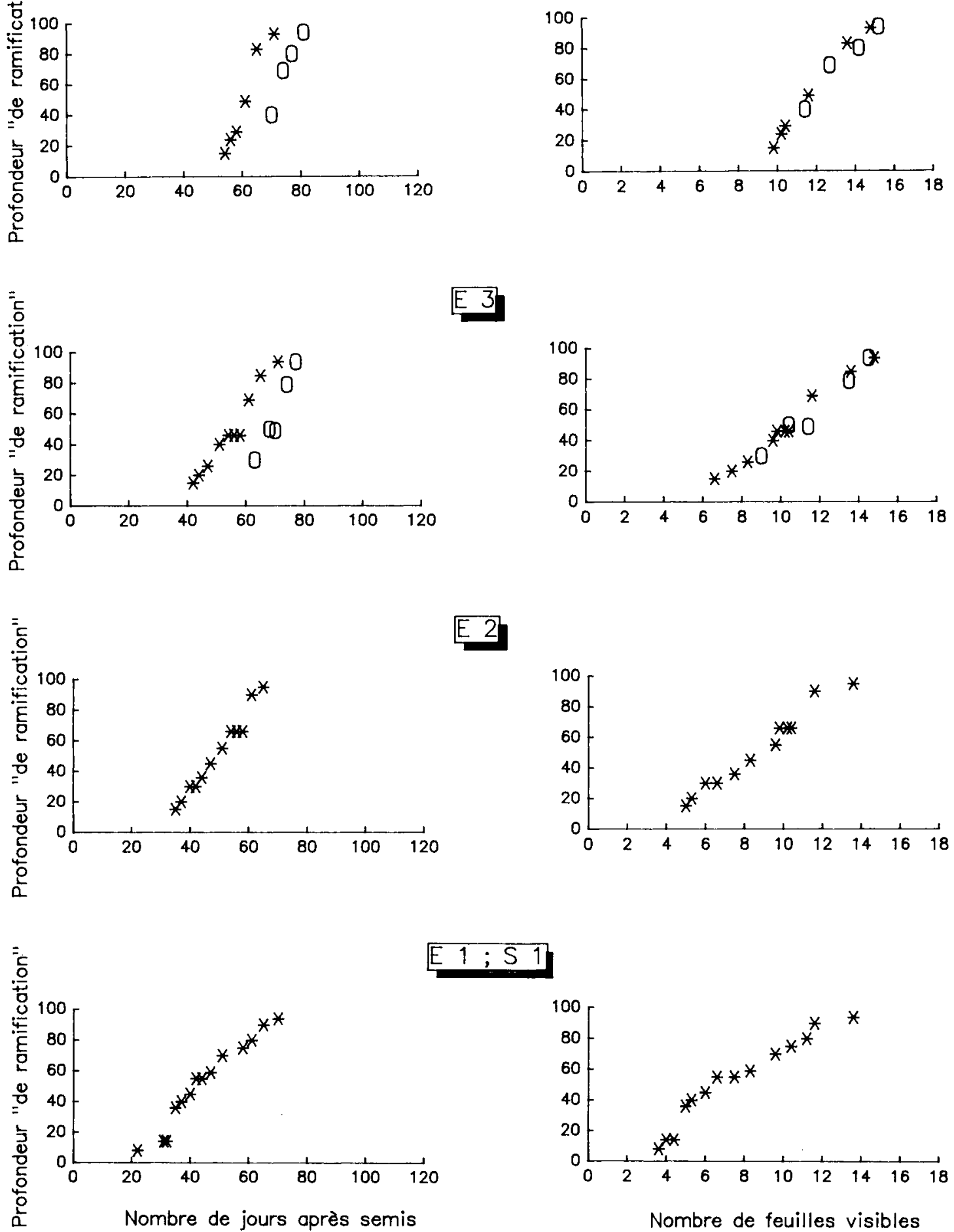

Fig 6. Cinétiques d'émission des racines secondaires pour chacun des entre-nœuds pour les 2 années d'étude, en fonction du

riyama (1983) et sur riz par Kawata et al (1980). D'une manière générale, les nombres totaux (i) de ramifications et de ramifications allongées par unité de longueur, et de (ii) primaires par entrenœud (Picard et al, 1985) augmentent avec le rang d'insertion de $E_{i}$ sur la tige. En consé- 
b
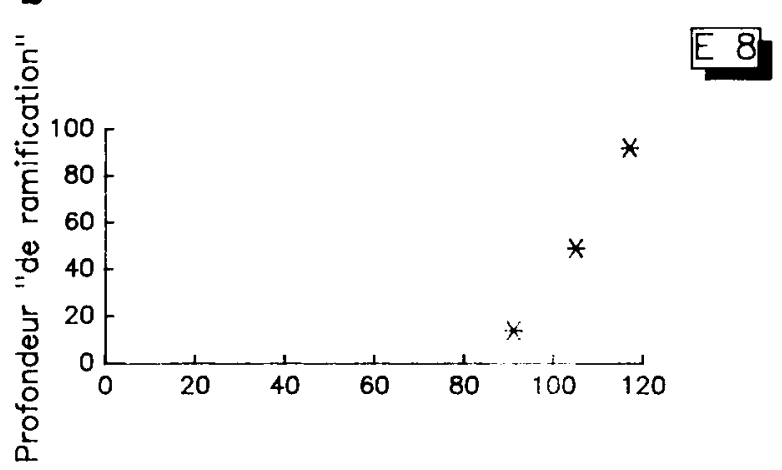

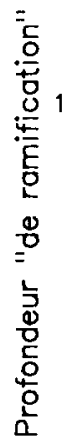
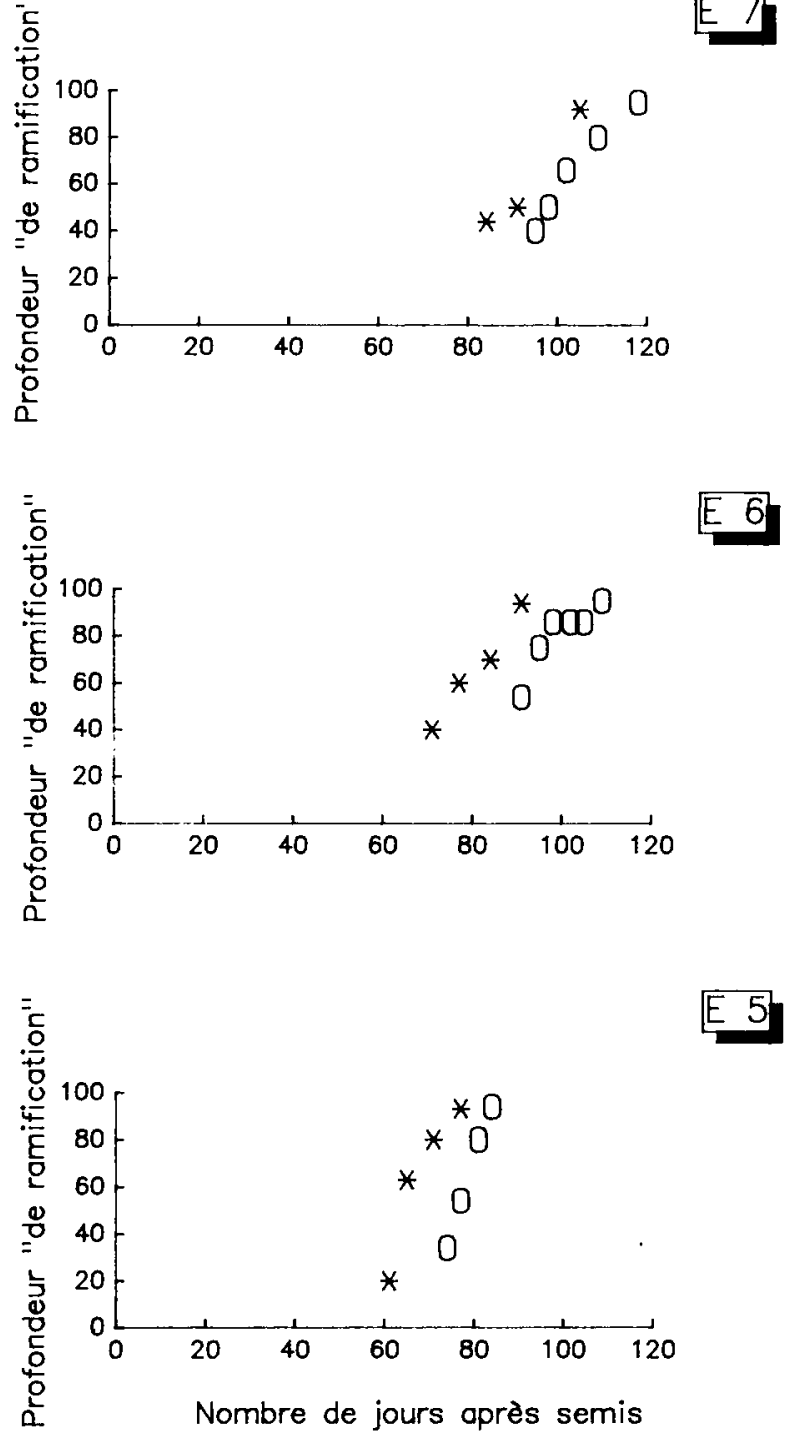

Flg 6b. Cinétiques d'émission des racines secondaires pour chacun des entre-nœuds pour les 2 années d'étude, en fonction du nombre de jours après semis et du nombre de feuilles visibles. Entre-nœeuds de 5 à 8 .
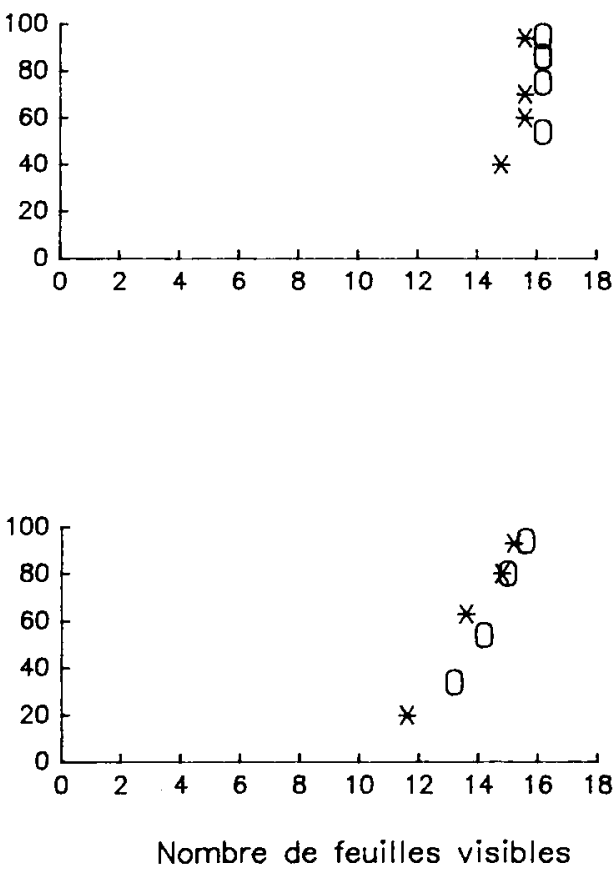

nombre de jours après semis et du nombre de feuilles visibles; a : entre-nœuds $1-4$. b : entre-nœuds 5-8.

quence, la cinétique d'émission et d'élongation des racines d'ordre 2 (évolution des nombres de secondaires totales et allongées) est de type exponentiel.

La variabilité importante des mesures inter- et intra-plante est liée :
- pour les secondaires totales, à leur disposition aléatoire sur les primaires (Girardin, comm pers). Chez le maiis, le nombre de sites de ramification (génératrices correspondant aux pôles ligneux des racines d'ordre 1 (Camefort, 1972), généralement corrélé au diamètre de la primaire 
Tableau III. Durée de l'émission secondaire, sur les différents entre-nœuds, en deçà de $80 \mathrm{~cm}$ de profondeur.

Entre-nœud

Émission des secondaires

Date de début

Apparition des premières secondaires sur les portions de primaires visibles à travers la vitre du rhizotron
Date de fin

Plus d'émission de secondaires sur les portions de primaires visibles à travers la vitre du rhizotron

En nombre de jours après le semis

$\begin{array}{cc}S+E_{1} & 23 \\ E_{2} & 37 \\ E_{3} & 42 \\ E_{4} & 54 \\ E_{5} & 61 \\ E_{6} & 71 \\ E_{7} & 84 \\ E_{8} & 91\end{array}$

$\begin{array}{rr}61 & 38 \\ 71 & 34 \\ 71 & 29 \\ 71 & 17 \\ 77 & 16 \\ 91 & 20 \\ 105 & 21 \\ 117 & 26\end{array}$

En nombre de feuilles visibles

$\begin{array}{cr}S+E_{1} & 3,7 \\ E_{2} & 5,3 \\ E_{3} & 6,7 \\ E_{4} & 9,8 \\ E_{5} & 11,5 \\ E_{6} & 14,8 \\ E_{7} & \\ E_{8} & \end{array}$

$\begin{array}{rr}11,5 & 7,8 \\ 13,6 & 8,3 \\ 13,6 & 3,8 \\ 13,6 & 3,8 \\ 15,6 & 4,1 \\ >15,8 & >1\end{array}$

Fin de l'émission foliaire

(Torrey et Wallace, 1975) est très supérieur à celui des secondaires par unité de longueur. II est en moyenne égal à 16 pour $E_{2}$ et à 51 pour $E_{6}$ (résultats non publiés). Ceci permet une certaine souplesse quant à la localisation des initiums racinaires dont l'alignement sur les génératrices précitées est irrégulier (Bell et Mc Cully, 1970);

- pour les secondaires, de plus de $3 \mathrm{~cm}$, à l'influence de la position de chaque ramification au sein du système sur son allongement éventuel. $\mathrm{La}$ variation des nombres moyens de racines longues en fonction de l'année tend à montrer que la configuration spatiale des réseaux est déterminée préférentiellement par les conditions de milieu, pris au sens large (cf paragraphe suivant). Cette régulation de la forme par le devenir des secondaires semble être spécifique au maïs. En effet, chez les autres graminées cultivées elle se traduit principalement par une modification importante des nombres de racines primaires et secondaires, consécutive à un tallage plus ou moins important (Jordan, 1992b). Or, dans le cas du maïs, cette possibilité a été éliminée par la pression de sélection qui a supprimé le tallage.

L'origine du mécanisme de différenciation et d'élongation d'une secondaire sur un site donné n'est pas connue. Mais plusieurs pistes paraissent devoir être explorées : il nous faut quantifier l'influence du sol qui intervient localement au travers de ses propriétés physiques (primaires coudées et portant des secondaires longues aux points d'inflexion car ne pouvant pénétrer les agrégats, souvent en raison d'un stress hydrique important (Phillips et Kirkham, 1962; Nye et Tinker, 1977) et chimiques. On sait, par exemple, que les nombres de ramifications dépendent de la biodisponibilité de l'azote (May et al, 1965; Brouwer, 1981; Geisler et Krutzfeldt, 1983); 
- l'éventualité d'une «régulation trophique» peut également être envisagée : les taux d'émission et de croissance sont fonction de la quantité d'assimilats disponibles. Au sein du système racinaire ceux-ci peuvent être répartis soit de façon aléatoire, soit en privilégiant certains entre-nœuds ou ordres racinaires de façon à permettre la colonisation préférentielle d'horizons de sol prédéterminés;

- signalons enfin que la variabilité observée peut avoir une cause plus physiologique. En effet, compte tenu du fait qu'une primaire se ramifie au fur et à mesure de sa croissance, il est possible que les nombres de secondaires émises et allongées soient d'autant plus faibles que l'élongation de la primaire porteuse est rapide. En effet, si la vitesse de croissance d'une racine d'ordre 1 est importante, il est possible que le temps imparti à la différentiation des initiums de racines secondaires et à leur allongement ultérieur soit trop court pour permettre l'expression de la totalité du potentiel de ramification. Dans ce cas il se pourrait que, comme cela a été observé pour les racines d'ordre 1 (Girardin et al, 1987), toutes les ébauches initiées (amas de quelques cellules différenciées) ne soient pas émises, c'est-à-dire ne percent pas l'épiderme de la primaire.

Ces différentes hypothèses ne peuvent être confirmées (ou infirmées) par des études complémentaires que si l'on connaît au préalable les mécanismes qui permettent la différentiation de ces 2 catégories de secondaires. II nous faut, en particulier, préciser le stade auquel se produit l'arrêt de croissance, savoir s'il est le même pour tous les organes concernés, donc si, d'un point de vue histologique, les secondaires courtes ont toutes la même structure. Si tel est le cas, le comportement d'une ramification vis-à-vis de l'allongement est déterminé de façon précoce, au moment de la différentiation des tissus. Des observations de coupes transversales portant sur la proportion relative des différents tissus et sur la répartition et la taille des vaisseaux du xylème devraient apporter quelques éléments de réponse quant aux modalités de cette sélection par rapport à l'allongement et le rôle, pour la survie de la plante des racines courtes.

Notons cependant que, si une réduction d'environ $15 \%$ du nombre de ramifications (cas de la parcelle Flaxlanden-1983) ne pénalise pas la production de biomasse aérienne, il est fortement probable qu'en conditions non limitantes, la contribution des secondaires courtes à la nutrition de la plante soit négligeable.

\section{La cinétique d'émission}

Les lois de ramification que nous avons mises en évidence sont relativement plus complexes que celles décrivant l'émergence des primaires (Picard et al, 1985) en raison du double déterminisme que suit l'émission racinaire :

- la date d'apparition des premières racines d'ordre 2 sur un réseau de rang $E_{i}$ peut être déduite du phyllochrone. Ceci semble être un comportement général aux graminées (Yamazaki et Harada, 1982; étude sur riz; Klepper et al, 1984 , étude sur blé);

- la progression du front de ramification le long d'une primaire dépend de sa vitesse de croissance. Le pédo-climat a une influence prépondérante sur ce paramètre; il intervient au travers de la température, du potentiel matriciel du sol et de la vitesse de diffusion de l'oxygène (Wang, 1983).

Nos résultats montrent par ailleurs que les cinétiques de mise en place des 2 ordres racinaires ne sont pas identiques. En effet, la durée, mesurée en phyllochrone, qui sépare l'émission sur 2 entre-nœuds successifs est plus importante pour les secondaires $(2,20)$ que pour les primaires (2,02; Picard et al, 1985). En conséquence, l'écart entre l'apparition des racines d'ordre 1 et celles d'ordre 2 sur un réseau $E_{i}$ s'accroît légèrement au cours du cycle.

Notons enfin que l'émission des premières secondaires sur des primaires issues d'un entrenœud donné ne modifie ni les vitesses d'élongation, ni les taux de ramifications (nombres d'organes émis) des primaires issues des entrenœuds inférieurs ( $c f$ fig 6 ). La question se pose donc de savoir à partir de quelle profondeur (qui ne peut qu'être supérieure à $90 \mathrm{~cm}$, étant donné la taille des rhizotrons), la croissance et la ramification d'un réseau sont ralenties, et quel est le déterminisme de ce comportement : diamètre des vaisseaux conducteurs trop faibles pour assurer une alimentation correcte, longueur des primaires limitées par un contrôle hormonal.

\section{RÉFÉRENCES}

Bell JK, Mac Cully ME (1970) A histological study of lateral root initiation and development in Zea mays. Protoplasma 70, 179-205

Blacklow WM (1972) Influence of temperature on the elongation of radicle and shoot of corn (Zea mays L). Crop Sci $647-650$ 
Bohm W (1979) Methods for studying root systems. Ecological Studies 33, Springer Verlag, $188 \mathrm{p}$

Brouwer R (1981) Co-ordination of growth phenomena within a root system of intact maize plants. In: Structure and function of plant roots (Brouwer $\mathrm{R}$, Gasparikova O, Kolek J, Loughman BC, eds) Junk, La Haye, 269-273

Camefort H (1972) Morphologie des végétaux vasculaires. Cytologie. Anatomie. Adaptations. Doin, Paris, $405 \mathrm{p}$

Cooper AJ (1973) Root temperature and root growth. Commonwealth Agricultural Bureau, Slough, UK, $73 \mathrm{p}$

De Roo HC (1957) Root growth in Connecticut tabacco soils. Conn Agric Exp Stn Bull 608

Diggle AJ (1988) ROOTMAP. A model in threedimensional coordinates of the growth and structure of fibrous root systems. Plant Soil 105, 169-178

Franquin $P$ (1974) Un modèle théorique du développement de la structure de la plante. Physiol Vég $12,459-465$

Geisler G, Krutzfeldt B (1983) Untersuchungen zur Wirkung von "Stickstoff" auf die Morphologie, die Trokenmassebildung und die Aufnahmeleistung der Wurtzelsysteme von Mais, Sommergersten-, und Ackerbohnensorten unter Berucksichtigung der Temperatur. I. Wurtzerlmorphologie. Z Acker Pflanzenbau 152, 336-353

Girardin P, Jordan MO, Picard D, Trendel R (1986) Harmonisation des notations concernant la description morphologique d'un pied de maïs (Zea mays L). Agronomie 6, 873-875

Girardin P, Morel-Fourrier B, Jordan MO (1987) Développement des racines adventives chez maïs. Agronomie 7, 695-702

Gounot M, Atry M, N'Kanda J, Yu O (1980) Photosynthèse nette et rythme d'apparition des feuilles chez le dactyle (Dactylis glomerata L). CR Acad Sci Sér D: Sci Nat 290, 1257-1260

Grimes DW, Miller RJ, Willey Pi (1975) Cotton and corn root development in two field soils of different strength characteristics. Agron J 67, 519-523

Habib R, Pagès L, Jordan MO, Simmoneau T, Sébillotte $M$ (1991) Approche à l'échelle du système racinaire de l'absorption hydro-minérale. Conséquence en matière de modélisation de l'absorption racinaire. Agronomie 11, 623-643

Jordan MO (1986) Mise en place du système racinaire du maïs. Définition et caractérisation au champ et en conditions contrôlées des paramètres descriptifs de la structure racinaire. Construction de l'organigramme d'un modèle cinétique. Thèse (Doctorat d'université), université Louis Pasteur, Strasbourg, $177 \mathrm{p}$

Jordan MO (1992a) Les rhizotrons peuvent-ils être utilisés pour l'étude de la ramification secondaire du maïs (Zea mays L) ? Agronomie 12, 3-14

Jordan MO (1992b) Relations entre le développement des parties aériennes et racinaires au niveau d'un peuplement. In: Le point sur les racines. Publication INRA (à paraître)

Kawata S, Sasaki O, Yamazaki K (1980) On the lateral root formation in relation to the diameter of crown roots in rice plants. Jpn J Crop Sci 49, 103-111

Klepper B, Belford RK, Rickman RW (1984) Root and shoot development in winter wheat. Agron $J 76$, 117-122

Lambers H, Posthumus $F$ (1980) The effect of light intensity and relative humidity on growth rate and root respiration of Plantago lanceolata and Zea mays. $J$ Exp Bot 31, 1621-1630

Lungley DR (1973) The growth of root systems. A numerical computer simulation model. Plant Soil 38, 145-159

May LH, Chapman FH, Aspinall D (1965) Quantitative studies of root development. I. The influence of nutrient concentration. Aust J Biol Sci 18, 25-35

Mirreh HF, Ketcheson JW (1973) Influence of soil water matric potentiel and resistance to penetration and corn root elongation. Can J Soil Sci 53, 383-388

Nelson WW, Allmaras RR (1969) An improved method for excavating and describing roots. Agron $J 61$, $75 \uparrow-755$

Nye PH, Tinker PB (1977) Solute movement in the soil-root system. Blackwell Sci Publ, Oxford, $342 p$

Pagès L, Aries F (1988) SARAH : modèle de simulation de la croissance, du développement et de l'architecture des systèmes racinaires. Agronomie 8, 889-896

Phillips RE, Kirkham D (1962) Mechanical impedance and corn seedling root growth. Soil Sci Soc Am Proc 26, 319-322

Picard D, Jordan MO, Trendel R (1985) Rythme d'apparition des racines primaires du maïs (Zea mays L). I. Étude détaillée pour une variété en un lieu donné. Agronomie 5, 667-159

Torrey JC, Wallace WD (1975) Further studies on primary vascular tissue pattern formation in roots. $I n$ : The development and function of roots (Torrey $\mathrm{JC}$, Clarkson DT, eds) Acad Press, London, 618 p, 91 103

Torrey JC (1976) Root hormones in plant growth. Annu Rev Plant Physiol 27, 435-459

Wang $J$ (1983) Growth simulation of the corn radicle root for various soil physical conditions. PHD, Pennsylvania State Univ, Dissertation Abo, Int Section B

Wightman F, Schneider EA, Thimann KV (1980) Hormonal factors controlling the initiation and development of lateral roots. 2. Effects of exogenous growth factors on lateral root formation in pea roots. Physiol Plant 49, 304-314

Yamazaki K, Harada J (1982) The root system formation and its possible bearings on grain yield in rice plants. Jpn J Crop Sci 15, 153-160

Yamazaki K, Kaeriyama N (1983) The diameter of primary roots and the lateral root formation in corn plants. Jpn J Crop Sci 52, 59-64 